山्山FFANÇAISE

$\supset \mathrm{DE}$

$\stackrel{1=1}{\simeq}$ PÉDAGOGIE

\section{Revue française de pédagogie}

Recherches en éducation

182 | 2013

Connaissances et politiques d'éducation : quelles interactions?

\title{
Le rôle des connaissances dans le champ de l'éducation belge francophone : de la nécessité de discrétion au développement hétéronome de connaissances
}

The role of knowledge in the French-language Belgian field of education. From required discretion to the heteronomous development of knowledge

Bernard Delvaux et Éric Mangez

\section{(2) OpenEdition}

Édition électronique

URL : http://journals.openedition.org/rfp/3994

DOI : $10.4000 /$ rfp.3994

ISSN : 2105-2913

Éditeur

ENS Éditions

Édition imprimée

Date de publication : 28 août 2013

Pagination : $31-40$

ISSN : 0556-7807

\section{Référence électronique}

Bernard Delvaux et Éric Mangez, «Le rôle des connaissances dans le champ de l'éducation belge francophone : de la nécessité de discrétion au développement hétéronome de connaissances », Revue française de pédagogie [En ligne], 182 | 2013, mis en ligne le 28 août 2016, consulté le 01 mai 2019. URL : http://journals.openedition.org/rfp/3994; DOI : 10.4000/rfp.3994 


\section{Le rôle des connaissances dans le champ de l'éducation belge francophone : de la nécessité de discrétion au développement hétéronome de connaissances}

\section{Bernard Delvaux et Éric Mangez}

La sociologie nous invite à considérer les connaissances comme tributaires de leur contexte et de leurs conditions (sociales, politiques, pratiques, etc.) de production. Sociologiser ainsi les connaissances et les manières de connaître signifie aussi que l'usage et l'utilité des connaissances pour et par le politique sont susceptibles de subir des variations selon les contextes. Dans cette contribution, nous commençons dès lors par rappeler les spécificités du contexte social et politique belge, pour en inférer certains enseignements relatifs à la place des connaissances dans la construction des politiques publiques. Nous développons ensuite cette problématique en nous concentrant sur le secteur de l'éducation dans la partie francophone du pays et en distinguant les héritages issus d'une histoire longue et certaines transformations récentes. L'analyse montre en effet une relative rupture dans l'histoire entre un passé toujours présent qui promeut la discrétion et un à-venir déjà là qui impose au champ de l'éducation des critères de connaissance et d'évaluation sur lesquels les acteurs du champ ont une emprise limitée.

Mots-clés (TESE) : Communauté Française de Belgique, politique en matière d'éducation, décideurs en matière de politique éducative, système éducatif.

L'organisation politique de la Belgique dans son ensemble présente d'importantes particularités qu'il est nécessaire de prendre en compte pour saisir le rôle des connaissances dans ce contexte (Mangez, $2009,2010)^{1}$. Nous n'allons pas procéder ici à une description systématique des institutions politiques qui composent le paysage belge, mais plutôt évoquer quelques traits saillants de la culture politique : les manières de faire, les habitudes, voire les habitus politiques qui président à la construction des politiques publiques en Belgique. Après avoir présenté la situation qui prévaut jusqu'au seuil des années 1990, nous nous tournerons vers les transformations récentes.

\section{CONNAISSANCE ET POLITIQUE EN BELGIQUE JUSQU'AU SEUIL DES ANNÉES 1990}

La culture politique belge s'est constituée dans une histoire longue. On sait qu'une des principales conditions de possibilité de la Belgique comme ÉtatNation a consisté en la construction, dès 1830-1831, d'un accord fondamental entre deux communautés (catholique et anticléricale) qui n'ont accepté de vivre ensemble qu'à la condition de se voir accorder un certain nombre de libertés, notamment pour organiser leur vie collective (Mabille, 2000). Ceci constitue un acte fondateur bien connu, qui établit les bases d'une relation spécifique entre l'État et une " société civile 
organisée » (Reman, 2002) au sein de laquelle cohabitent différentes communautés. Dans une diversité de secteurs, chacune de ces communautés a alors mis en place un certain nombre d'organisations qui, ensemble, constituent ce que les intellectuels belges et néerlandais ont pris l'habitude de nommer un pilier (Seiler, 1997 ; Vanderstraeten, 2002) ou un segment (Lijphart, 1979, 1985). Le concept de pilier - et le processus de pilarisation - sert à désigner ce processus au travers duquel une communauté culturelle organise sa vie collective en mettant en place des organisations actives dans des champs différents. On trouve ainsi, au sein du monde laïc (dans sa branche socialiste davantage que dans sa branche libérale), de nombreuses organisations : hôpitaux, mouvement de jeunesse, syndicats, universités, mutualité, coopératives dans le domaine des assurances et des banques, etc. De même, du côté catholique, on trouve une grande variété d'organisations actives dans une diversité de champs (santé, éducation, culture, enseignement supérieur et recherche, loisirs, mouvement de jeunesse, secteur bancaire, assurances, etc.) et constitutives du pilier chrétien (Jelen \& Wilcox, 1998). Cette pilarisation s'est développée avec davantage de succès dans les secteurs de service (éducation, santé, loisirs, syndicalisme, etc.) que dans des secteurs moins personnalisés (finance, industrie, etc.) (Vanderstraeten, 2002). S'il est évident que les piliers ont perdu une partie de leur solidité institutionnelle, s'il est évident également qu'on assiste aujourd'hui à une forme de dissociation entre les organisations propres aux piliers et leurs bases sociologiques (De Munck, 2002), il n'en demeure pas moins qu'ils ont donné une certaine épaisseur et une certaine consistance à la culture politique belge.

\section{Culture politique et partage du pouvoir dans une société segmentée}

Cette culture s'est construite sur le principe selon lequel il faut trouver des manières d'agir qui permettent à des groupes différents de vivre ensemble tout en préservant, au moins en partie, leur autonomie d'existence collective. Ces manières d'agir qui permettent le vivre ensemble dans une société segmentée sont par exemple le scrutin proportionnel (plutôt que majoritaire), l'octroi et le maintien d'une certaine autonomie aux acteurs collectifs organisés (notamment au travers de libertés constitutionnelles), le financement par les pouvoirs publics des activités organisées par ces collectifs lorsqu'elles sont considérées d'utilité publique (selon le principe de la liberté subsidiée par exemple), la négociation de différentes formes de «pactes » (scolaire, culturel, associatif) supposés garantir un partage du pouvoir social et symbolique. On peut ainsi comprendre par exemple pourquoi les pouvoirs publics belges définissent aujourd'hui leur relation avec le monde associatif comme une relation de “complémentarité ». Cette manière de poser les relations entre l'État et la société, qui est fort différente du modèle français, doit être prise en compte dans l'analyse des rapports entre connaissance et politique.

La cohabitation démocratique de différentes communautés sur un même territoire ne peut se réaliser qu'à certaines conditions, qui sont celles de la démocratie consociative (Lijphart, 1979 ; Bakvis, 1985 ; Byrne, 2001). La construction des politiques publiques dans ce type de démocratie se caractérise en effet par différents traits, que divers analystes de la vie politique et sociale belge ont bien identifiés :

- en général, l'État ou, plus largement, les pouvoirs publics sont relativement faibles et " mis en position de subordination par rapport à [la] société civile organisée » (De Munck, 2002) ;

- les savoirs pertinents dans la plupart des secteurs de l'action publique ne se situent pas dans les administrations officielles de l'État (De Munck, 2002) ;

- l'autonomie segmentaire des acteurs organisés est importante et prévaut à défaut d'accord intersegmentaire (Seiler, 1997) ;

- les politiques publiques se construisent du «bas vers le haut ", ou plus précisément des piliers vers le " centre ", au travers de la fabrication de compromis inter-segmentaires entre acteurs organisés, quasi institués (Dumont \& Delgrange, 2008).

La caractéristique essentielle de ce type de modèle démocratique tient à la place accordée aux acteurs organisés situés " entre » les pouvoirs publics et les citoyens individuels. Dumont et Delgrange (2008) synthétisent bien certains aspects du modèle de démocratie à l'œuvre en Belgique, notamment en soulignant le contraste avec la France. Le modèle républicain français " est conçu selon un mouvement déterminant qui procède du haut vers le bas, de l'État vers la société, de la politique vers la culture ". À l'inverse, en Belgique, “ [au] lieu de partir de l'État pour descendre dans la société, la culture politique [se] construit à partir du bas, de la pluralité des traditions religieuses, idéologiques et régionales, pour remonter vers le politique et l'État [...]. [La] réalité sociopolitique du système belge de la décision publique est caracté- 
risée de manière massive par la logique de la négociation et du compromis " (Dumont \& Delgrange, 2008, p. 82-83). Les mêmes auteurs précisent que, dans le contexte belge, "les autorités publiques doivent intervenir de manière positive pour aider les diverses tendances idéologiques et philosophiques représentatives à développer leurs activités d'utilité publique, et les associer à l'élaboration des politiques et à la gestion des services publics relevant de leur domaine d'intervention » (Dumont \& Delgrange, 2008, p. 82).

Si la culture politique suit des chemins si différents selon que l'on se situe dans un contexte ou un autre (en France ou en Belgique par exemple), on est en droit de penser que le rôle qu'y joue le savoir de même que l'articulation entre savoir et pouvoir se déclinent de manière différente également.

\section{Place des savoirs et principe de discrétion}

La manière "belge » d'organiser une forme de cohabitation pacifique entre groupes est longtemps allée de pair avec un principe de discrétion. Cette nécessité de discrétion doit être entendue comme une habitude de prudence ou de diplomatie qui, dans le contexte consociatif (Lijphart, 1979), est porteuse d'une utilité politique fondamentale : elle met en acte l'autonomie segmentaire et, en même temps, elle pose en puissance le compromis inter-segmentaire. La notion de discrétion s'entend alors au double sens du terme : il s'agit à la fois de laisser un certain nombre de choix à la discrétion des acteurs organisés (ce qui garantit leur autonomie en pratique), mais aussi de maintenir une certaine discrétion au sens où l'on ne se mêle pas des affaires de son voisin (ce qui rend le compromis possible en puissance).

On peut comprendre, à partir de ces éléments, le constat posé par Varone et ses collègues (Varone \& de Visscher, 2001 ; Varone \& Jacob, 2004) pour qui l'évaluation des politiques publiques a tendance à être " plus institutionnalisée dans les démocraties majoritaires (soit le modèle de Westminster ou un régime présidentiel) que dans les démocraties dites de consensus, de négociation ou de concordance ". Le développement de l'évaluation des politiques publiques se réalise en effet plus lentement et plus difficilement au sein des démocraties consociatives, notamment parce qu'un tel développement risque de nuire à l'autonomie des différents acteurs et parce qu'elle n'est pas perçue comme un élément nécessaire à la construction de compromis. Au sein d'une démocratie consociative, dans le travail de construction de politiques publiques, il ne s'agit pas tant de
" trancher » entre des alternatives que d'« assembler » des idées et des intérêts parfois en tension. Si la mobilisation de connaissances n'est pas en soi nécessairement contradictoire avec cette culture politique, elle ne paraît pas non plus s'y inscrire avec la force de la nécessité. On doit par ailleurs souligner que, dans ce type de démocratie, il n'est pas nécessairement possible d'identifier un acteur, même public, disposant de suffisamment de légitimité pour imposer des procédures d'évaluation des politiques publiques. Bien sûr, ce contexte n'empêche pas tel ou tel acteur collectif spécifique de développer en son sein une certaine culture de l'évaluation, mais il est peu favorable au développement généralisé d'une telle culture.

Ces différents éléments permettent aussi de comprendre pourquoi les administrations belges ont peu développé, en dehors des recensements décennaux, de grandes enquêtes et de larges bases de données, comme cela a été le cas dans d'autres pays. En effet, la maîtrise fine et complète de données statistiques ne constitue pas un ingrédient fondamental dans la construction des politiques dans un contexte où ces dernières sont presque nécessairement le résultat de transactions, de compromis, d'« arrangements " qui ne se réalisent pas en fonction d'une rationalité objectivante, mais bien en fonction d'une capacité et d'une habileté à produire des compromis que l'« évidence » de données objectivées ne favorise pas nécessairement. Les administrations n'ont dès lors guère accumulé d'expertise en leur sein. Celle-ci se situe traditionnellement ailleurs, et en particulier au sein de la société civile organisée (De Munck, 2002).

\section{Le champ de l'éducation au seuil des années 1990}

Au seuil des années 1990, le champ éducatif belge francophone ressemblait au portrait général dressé plus haut. Le modèle était largement consociatif, les piliers se déclinant sous la forme de réseaux, avec, principalement, un réseau libre catholique renvoyant au pilier catholique et des réseaux officiels (pouvoirs locaux d'une part, pouvoir central de l'autre) renvoyant au pilier laïc ${ }^{2}$. Les syndicats enseignants, puissants et bien implantés, étaient eux-mêmes associés aux piliers (même si les syndicats socialistes et libéraux avaient une centrale professionnelle pour l'enseignement catholique et si le syndicat chrétien en avait une dans l'enseignement officiel). En dépit de l'existence d'un ministère et d'un ministre de l'Éducation, en dépit également du subventionnement public de la majeure partie des dépenses de l'enseignement libre, une part importante des compétences décisionnelles était du 
ressort des pouvoirs organisateurs ou de leurs fédérations, ces dernières jouant un rôle plus important dans le réseau catholique que dans le réseau des pouvoirs locaux, d'abord sous la forme des congrégations, ensuite sous la forme des diocèses puis du secrétariat général de l'enseignement catholique.

Le lent processus de communautarisation qui aboutit à la fin des années 1980 n'est pas un facteur de bouleversement des équilibres du champ éducatif, mais il introduit un clivage supplémentaire. Depuis le 1 er janvier 1989, les Communautés flamande, française et germanophone se sont en effet substituées à l'État central et gèrent le secteur de l'enseignement sans droit de regard des autorités fédérales, lesquelles n'ont conservé que trois prérogatives (fin et début de l'obligation scolaire, conditions minimales de délivrance des diplômes et régimes des pensions). Le secteur de l'enseignement est ainsi clairement cloisonné en trois entités autonomes, la Communauté française regroupant $42 \%$ des effectifs scolaires ${ }^{3}$.

Ces entités vivront, durant les vingt années suivantes, de manière nettement indépendante, sans pratiquement aucune concertation entre elles si ce n'est pour négocier, à l'échelle fédérale, des clés de répartition de leur financement ${ }^{4}$. Elles forment en réalité trois champs de l'éducation distincts, chacun caractérisé par un régime clairement consociatif avec cependant des rapports de force très différents entre piliers, le pilier catholique, pourtant fort bien implanté en Communauté française, étant encore nettement plus présent en Communauté flamande. Ainsi, chacun des deux principaux champs de l'éducation (le néerlandophone et le francophone) était alors à la fois peu autonome, en particulier vis-à-vis du champ du pouvoir (où interagissent les acteurs dominants issus des piliers), fragmenté et marqué par une nécessité de discrétion.

\section{LES TRANSFORMATIONS RÉCENTES}

Dans ce paysage communautarisé (et qui va, sur ce plan, demeurer inchangé au cours des vingt années suivantes), le modèle consociatif et la relative discrétion inter-segmentaire qui lui est associée vont être progressivement remis en cause par des processus essentiellement à l'œuvre à des échelles supranationales.

\section{Évolutions supranationales : opportunité et défi pour l'autorité publique}

Quatre évolutions, qui dépassent le contexte belge, ont significativement affecté le modèle consociatif. La première est la sécularisation générale des sociétés. Cette évolution est une source potentielle d'affaiblissement des piliers qui, en Belgique, se sont constitués autour du clivage catholique/laïc. L'affaiblissement de la "société civile organisée " constitue aussi une opportunité pour les autorités publiques de reprendre le pouvoir sur certains champs - en particulier celui de l'éducation. La seconde évolution est de nature technologique : c'est l'évolution rapide des technologies de l'information, qui présentent une menace potentielle pour la tradition de discrétion et pour l'absence relative de développement des bases de données. La troisième évolution résulte de la mondialisation et consiste en une interdépendance accrue entre acteurs et entre champs, qui sont tout à la fois contraints à la coopération et impliqués dans des relations de compétition, ce qui remet potentiellement en cause l'étanchéité des cloisonnements entre piliers, entre Communautés ou entre secteurs de l'action publique. Enfin, en lien direct avec ce développement des liens d'interdépendance, on assiste à la multiplication d'instances de coordination à l'échelle supranationale, ainsi que d'agences et d'ONG internationales. Ces évolutions elles aussi remettent en question les cloisonnements aussi bien que le principe de discrétion.

Ces diverses tendances sont de nature à remettre en cause certains aspects du modèle qui s'était sédimenté en Belgique, en particulier dans le secteur éducatif de la Communauté française. Elles vont de fait contribuer à repositionner l'acteur « étatique ». Les transformations du contexte international influent en effet sur la manière dont les États-Nations et les autres autorités publiques se pensent dans le monde. II ne s'agit plus tant pour eux de se construire et d'exister comme des acteurs nationaux, mais plutôt de s'engager dans une dynamique internationale qui les situe au sein de différents espaces de positions relatives dont les coordonnées sont souvent définies par des indicateurs de performances. L'espace national, communautaire ou régional dans le cas belge, ne constitue plus un espace à l'échelle duquel on peut penser de manière autonome les politiques publiques et la dynamique de leur fabrication. Le développement exponentiel des indicateurs transnationaux comparant les performances des États ou des régions est à la fois le fruit et le vecteur de cette perte d'autonomie. Les organisations internationales sont des acteurs majeurs de cette évolution. Dans le secteur éducatif, l'OCDE a 
joué en la matière un rôle de premier plan en étant le porteur du projet PISA consistant à évaluer les performances des systèmes éducatifs nationaux (Carvalho, 2009 ; Mangez \& Hilgers, 2012).

Pour la jeune autorité publique qu'est la Communauté française, cette tendance constitue à la fois une opportunité et un défi. Alors qu'elle occupait jusqu'alors une place limitée dans la régulation du champ de l'éducation, il est en effet désormais attendu de cette autorité publique qu'elle assume une part de la responsabilité du (bon) positionnement du champ dans la compétition. C'est clairement une opportunité pour affirmer son rôle. Dans le même temps, cette « mission » met en cause les divers cloisonnements, à commencer par le cloisonnement sectoriel. On assiste en réalité à un affaiblissement de l'autonomie relative des champs (Lemieux, 2011). L'éducation devient un secteur-clé dans la compétition économique, les discours en termes de capital humain et de société de la connaissance invitant à mettre l'éducation à son service. La mondialisation et la compétition internationale justifient le développement d'un nouveau rôle pour l'autorité publique en charge de l'enseignement en même temps qu'elles tendent à réduire son autonomie relative. Mutatis mutandis, c'est comme si l'autorité publique était appelée à assumer davantage son rôle de chef d'orchestre, mais aussi à faire jouer à l'orchestre une partition dont elle ne serait pas le compositeur mais seulement l'interprète.

De fait, la Communauté française assume davantage son statut de chef d'orchestre. On voit émerger peu à peu de nouveaux dispositifs de régulation à l'échelle communautaire : les uns prennent la forme de comités de concertation institutionnalisés associant les divers acteurs du champ, tous réseaux confondus (commission de pilotage, conseils centraux de concertation par niveau, etc.) ; d'autres se matérialisent sous les traits de services publics travaillant indifféremment dans tous les réseaux (inspection, service général du pilotage du système éducatif, etc.) ; d'autres enfin se concrétisent sous la forme de nouvelles normes légales transversales aux réseaux (en matière de statut des membres du personnel ou d'inscription des élèves notamment). Ainsi, l'autorité publique occupe une place plus grande qu'auparavant, au point qu'il devient davantage pertinent de parler d'un système scolaire plutôt que d'un champ fragmenté constitué de systèmes et sous-systèmes relativement indépendants. On voit également se développer les connaissances que cet acteur public détient sur le système : non seulement, avec le développement des TIC, les données augmentent considérablement en volume, mais leur fonction évolue, elles ne sont plus seulement utilisées à des fins de gestion administrative quotidienne mais servent aussi, et parfois prioritairement, à la régulation.

Pour autant, l'autorité publique communautaire ne devient pas un pouvoir centralisateur fort, à la fois pour des raisons historiques et parce qu'une telle centralisation serait en contradiction avec le nouveau modèle de la gouvernance. Le système éducatif belge francophone ne reprend de fait à son compte qu'une part des traits de ce nouveau modèle. Autrement dit, si les tendances lourdes au plan international s'imposent avec force en Belgique, la rhétorique de la gouvernance, que certains parviennent à imposer au plan international à la faveur de ces changements macrosociétaux, n'est pas toute puissante. Elle rencontre en effet un contexte national où l'histoire longue s'est cristallisée dans des cadres cognitifs et des organisations susceptibles de résister aux modèles importés. On verra dans les pages suivantes que le modèle de la gouvernance ne vient perturber qu'à la marge les modes d'interaction «locaux » entre les acteurs de la politique publique et ne parvient à imposer que sous une forme fortement hybridée le nouveau modèle de mobilisation des connaissances dans l'action publique. La raison en est que la société belge est en consonance avec le versant "multipolaire " du nouveau modèle, qu'elle n'a donc aucun mal à endosser, et en dissonance avec le versant « rationalisant » de ce même modèle, qu'elle n'adopte qu'en l'adaptant.

\section{Une société en consonance avec le modèle de l'action publique multipolarisée}

Si l'autorité publique communautaire ne devient pas un pouvoir centralisateur fort en dépit de son rôle croissant, c'est non seulement parce que, historiquement, le modèle politique belge est éloigné d'un tel modèle d'État centralisateur, mais aussi parce que les nouveaux modèles de régulation promus à l'échelle internationale sont ceux de la gouvernance. S'il n'est pas aisé de trouver une définition précise et stabilisée de la notion de "gouvernance ", il apparaît cependant clair qu'un de ses aspects centraux tient au fait qu'elle implique une diversité d'acteurs engagés dans une dynamique de fabrication de normes, de règles, de procédures. Elle désigne ainsi une manière de construire des politiques publiques qui se caractérise par le fait qu'elle inclut différents acteurs (publics, privés ou autres) et différents niveaux (européen, national, régional, local) dans le processus. 
La notion de gouvernance contraste ainsi avec une représentation - sans doute mythique, en particulier dans le cas belge - du travail politique comme étant le fait de l'autorité publique déployant sa force du haut vers le bas. Elle suppose en effet la prise en compte des intérêts et points de vue de cette diversité d'acteurs, mais aussi éventuellement leur implication dans le développement concret de politiques publiques. Les autorités publiques deviennent alors un acteur parmi d'autres. II ne s'agirait plus pour elles de « commander et contrôler " mais bien de consulter, de négocier, d'apprendre et de délibérer, puis de contractualiser, de piloter, d'évaluer, de subsidier, de coordonner. La gouvernance est ainsi présentée comme une pratique nouvelle en rupture avec une période passée durant laquelle l'État aurait été indissociablement plus fort et moins engagé dans différentes formes de relations avec les acteurs.

En Belgique francophone, ce «nouveau » modèle de gouvernance ne constitue pas vraiment une nouveauté. Bien plus, du point de vue de la diversité des acteurs impliqués, il y a concordance partielle entre ce "nouveau » modèle et les pratiques «traditionnelles " liées au régime consociatif. C'est donc dans ce modèle de gouvernance que vient se glisser assez naturellement l'autorité communautaire francophone.

Si cette autorité publique occupe une place plus importante qu'auparavant, elle s'insère dans une communauté d'acteurs plus qu'elle ne surplombe ces derniers. Poursuivant l'analogie du chef d'orchestre, nous dirons que la Communauté française se présente sous les traits d'un primus inter pares plutôt que d'un démiurge autoritaire et redouté. Certes, un certain nombre de compétences autrefois dévolues aux réseaux ou à leurs composantes (les pouvoirs organisateurs) tendent à être maintenant gérées en dehors des enceintes autrefois pilarisées et cloisonnées, mais, dans les instances qui héritent de ces nouvelles compétences, l'autorité communautaire partage souvent le pouvoir, voire le délègue. De fait, quand ces instances sont territorialisées à l'échelle des zones, l'autorité publique est assez rarement représentée. Quand elles se situent à l'échelle du système, ces instances prennent le plus souvent la forme de lieux de rencontre entre les différentes parties prenantes, dans lesquels le pouvoir communautaire joue davantage un rôle d'animateur que de décideur. Les piliers y conservent un pouvoir non négligeable et entrent souvent dans des logiques de compromis et de marchandage qui leur permettent à la fois d'infléchir les décisions et de préserver certaines de leurs prérogatives.
Beaucoup de ces lieux ne sont pas ouverts aux seuls organisateurs d'enseignement. On y retrouve souvent des représentants des syndicats d'enseignants ou des associations de parents, plus rarement des représentants d'étudiants ou des experts en éducation. Dans la grande majorité de ces lieux, les intervenants appartiennent exclusivement au champ éducatif, ce qui peut donner l'illusion d'un champ conservant une grande autonomie. De fait, l'institution "Église catholique » n'y est plus présente, et c'est encore de manière timide que les interlocuteurs sociaux y sont associés. Néanmoins, on observe que, d'une part, ces derniers tendent à investir les lieux de concertation relatifs à l'enseignement qualifiant ${ }^{5}$, réduisant de facto l'autonomie de ce "sous-champ ", et que, d'autre part, une partie des acteurs du champ tendent à reprendre à leur compte les discours d'acteurs externes à celui-ci, notamment en mettant en avant la fonction du système éducatif dans la compétition mondialisée et en portant le discours de la rationalisation de l'action publique, propre au modèle de la gouvernance.

\section{Une société en dissonance avec le modèle de l'action publique rationalisée}

$\mathrm{Si}$, sur le plan des modes d'interaction entre parties prenantes, le mode d'agir traditionnel belge est, comme on vient de le voir, relativement en adéquation avec le modèle de la gouvernance, et donc relativement peu mis en difficulté par lui, c'est nettement moins le cas sur le plan des usages des connaissances " pour " l'action publique. Sur ce plan, le modèle de la gouvernance se situe en rupture avec les pratiques héritées du passé. II promeut une rationalisation de l'action. Dans un tel modèle, la connaissance doit en effet permettre aux autorités de piloter et d'évaluer, mais aussi aux acteurs locaux d'être informés quant à leur contexte, aux « bonnes » pratiques et à leur capacité à atteindre un certain nombre d'objectifs ; bref elle doit contribuer à responsabiliser ces divers acteurs et les rendre soucieux de rationalité instrumentale.

Selon les pratiques et les discours normatifs à l'œuvre au niveau européen notamment, il s'agit désormais de promouvoir l'évaluation des politiques publiques, de développer l'evidence-based policy ou, dans une formule plus souple et plus récente, le knowledge-informed policy. II s'agit aussi d'observer et d'analyser les pratiques de ses voisins, proches ou lointains (benchmarking), en vue d'apprendre (policy learning) et d'identifier les bonnes pratiques (best practices). On le voit, le vocabulaire qui équipe ce modèle normatif est souvent anglo-saxon. 
En soi, l'histoire de la Belgique et la tradition de discrétion ne portaient pas en elles les germes de cette culture de l'évaluation et de la comparaison/ compétition. Nous ne nous situons pas non plus dans un contexte favorable au modèle de l'evidence-based policy ou à des pratiques de benchmarking. Ce sont des exigences extranationales, portées par le contexte mais aussi par des institutions européennes ou des agences intergouvernementales, qui vont peu à peu infléchir les pratiques héritées du passé, sans pour autant les transformer de manière radicale.

Indéniablement, depuis le début des années 1990, on assiste au développement des savoirs de gouvernement. On observe aussi une tendance au développement d'indicateurs et de dispositifs d'évaluation. Mais on constate également que : 1) ces savoirs ne sont pas seulement appropriés par l'autorité publique, mais aussi par les autres régulateurs centraux pilarisés, héritages de la société segmentée ; 2 ) ils n'impliquent pas une sortie de la logique de compromis ; 3) ceux qui les utilisent comme outils de régulation ne vont pas au bout de la logique de la responsabilisation et de la reddition de comptes.

La montée en puissance de ces connaissances s'est manifestée, à partir du milieu des années 1990, par la création de nouveaux services et la mise en œuvre de projets. Un service de pilotage, créé en 1996 au sein du ministère de la Communauté française, est devenu le principal artisan de la structuration de connaissances relatives à l'ensemble du système. Prudemment dénommé au départ «Service du pilotage inter-réseaux ", il a été par la suite significativement dénommé "Service général du pilotage du système éducatif ». Un service de traitement de l'information (ETNIC), constitué en 2002, a eu pour mission de structurer et de relier les bases de données individuelles sur les élèves, les enseignants et les établissements. Quant à la réforme des services d'inspection, mise en place en 2007 sans plus de référence au cloisonnement des réseaux, elle a conduit à une multiplication et à une systématisation des états des lieux du système.

Le changement ne concerne pas seulement le volume des connaissances mais aussi les finalités, la nature et le traitement des connaissances statistiques (Delvaux, Mangez C., Mangez É. et al., 2008). Les bases de données sont désormais utilisées à des fins de connaissance du système et plus seulement en vue de la gestion quotidienne des budgets, des enseignants, des élèves ou des établissements. Les statistiques, qui se résumaient initialement aux stocks (nombre d'élèves par année, sexe, région ou année de naissance), sont, dès 1994 et désormais de manière systématique, complétées par des données sur les résultats des élèves à des tests externes (Cattonar, Dumay, Mangez et al., 2010 ; Mangez C., 2010) et, plus récemment, par des statistiques des flux et des trajectoires d'élèves. Leur traitement a également changé, puisque la publication de données quasi brutes présentées dans de volumineux annuaires est désormais complétée par un système d'indicateurs mis en place après un long temps d'incubation.

L'autorité communautaire devient donc un acteur important de production de connaissances sur le système éducatif. Et les savoirs qu'elle produit sur le système contribuent à accroître la légitimité de ce système et de l'autorité en charge de sa coordination. Mais l'autorité communautaire n'est pas le seul acteur à développer de telles connaissances. À côté des acteurs du monde académique, les réseaux ont éprouvé eux aussi la nécessité de connaître leur propre système, aiguillonnés notamment par la montée des projets de connaissance au niveau du système global et soucieux de pouvoir résister aux volontés hégémoniques des tenants d'une régulation inter-piliers. Les réseaux n'ont cependant pas réagi de manière identique. Dans le pilier laïc, de tels savoirs se sont surtout constitués à l'échelle des (gros) pouvoirs organisateurs. Dans le pilier catholique, les connaissances se sont élaborées au niveau du réseau. Celui-ci réalise notamment des collectes de données et des enquêtes pour "objectiver » et pour " générer un processus de gestion de la connaissance qui dépasse l'intuition, le ressenti, le débat passionnel et idéologique " (un responsable du réseau catholique, entretien).

On assiste donc à une montée des savoirs de gouvernement sur l'éducation. De manière générale, le développement de connaissances peut contribuer au renforcement ou au déclin de l'autonomie d'un champ selon la manière dont les connaissances en question sont conçues et circulent. Un champ autonome est un champ qui produit sans cesse du savoir à son propre propos en fonction de ses objectifs spécifiques et qui conserve en son sein une part de ce savoir. À l'inverse, un champ peu autonome est un champ qui fait l'objet de mesures, d'évaluations, de production de connaissances selon des critères hétéronomes, définis voire produits par des acteurs externes au champ en question, et dont la circulation dépasse les frontières du champ. Qu'en est-il dans le champ éducatif francophone?

On constate d'une part que le champ tend à s'unifier, sa fragmentation s'atténuant en même temps que s'estompe la discrétion inter-segmentaire, sans toutefois s'effacer complètement puisque toute « réalité » n'est 
pas scrutable, toute connaissance n'est pas diffusable, et qu'il demeure malvenu de produire des savoirs qui mettent ouvertement en comparaison/compétition les différents réseaux. Mais, d'autre part, ce champ que constitue le système éducatif, tout en étant en formation du fait de ce processus de décloisonnement, se trouve lui-même en perte d'autonomie relative vis-àvis d'autres champs. Cela se traduit notamment au travers du développement de connaissances relatives à sa contribution à des impératifs externes au champ, en particulier sa contribution à la prospérité économique. Ces connaissances tendent aussi à mettre le champ de l'éducation francophone en compétition et en comparaison avec d'autres systèmes éducatifs, et en priorité avec la Communauté flamande, cet autre champ éducatif belge qui affiche des performances meilleures que son alter ego francophone, tant au plan éducatif qu'au plan économique. La production et la circulation des connaissances, auxquelles contribuent les pouvoirs publics, tendent donc, certes encore de manière limitée, à la soumission relative du champ éducatif au champ économique et, dès lors aussi à l'influence des meilleurs " compétiteurs ". Ainsi l'autonomie relative du champ éducatif aura-t-elle été de courte durée après son unification partielle par dé-segmentation et après le mouvement d'autonomisation de son segment catholique vis-à-vis de l'institution ecclésiastique (Delvaux, 2011).

\section{CONCLUSION}

Notre analyse montre que les quatre caractéristiques du régime consociatif, énoncées dans la première partie du texte, ont été partiellement remises en question suite aux évolutions supranationales décrites ci-dessus. Sur trois dimensions, on note une nette évolution. De fait, l'autorité publique est moins qu'avant mise en état de subordination. Elle détient bien plus qu'auparavant des savoirs pertinents pour la régulation du champ. De même, l'autonomie segmentaire des acteurs organisés s'est réduite significativement sans toutefois disparaître. Quant à la quatrième dimension (celle de la fabrication des politiques par compromis inter-segmentaire), elle a moins clairement évolué. La Belgique demeure en effet un pays fondamentalement basé sur la logique du compromis.
Quant au champ de l'éducation lui-même, on observe que le processus d'unification lié au déclin de la logique consociative qui s'est manifesté à la jonction du $x^{e}$ et du $x x^{e}$ siècle, et qui témoigne d'un gain d'autonomie à l'égard du clivage philosophicoreligieux (catholique/laïc), s'accompagne à la fois d'une perte d'autonomie à l'égard du champ économique et d'une nouvelle fragmentation. L'affaiblissement de l'autonomie relative du champ éducatif vis-à-vis des enjeux économiques s'opère moins par la présence d'acteurs externes au champ dans les instances de régulation que par l'intrusion plus discrète de référentiels externes, via les connaissances construites à propos de l'éducation. Ainsi, à la dynamique de gains d'autonomie vis-à-vis du clivage philosophicoreligieux et de pertes d'autonomie vis-à-vis des impératifs économiques, correspond, sur le plan des connaissances mobilisées dans l'action publique, un affaiblissement de la discrétion inter-segmentaire et le développement de connaissances hétéronomes.

Quant au processus de fragmentation, il suit deux logiques (Delvaux, 2011). D'une part, les domaines éducatifs les plus proches de l'emploi (enseignement qualifiant) se distinguent de plus en plus du reste du champ de l'éducation, tout en se rapprochant (dé-différenciation) du champ emploi-formation. D'autre part, le territoire bruxellois, lieu de cohabitation entre les Communautés flamande et francophone, tend à se constituer en un pôle revendiquant une spécificité et une autonomie partielle vis-à-vis des deux Communautés qui régissent chacune, sans concertation entre elles ni avec la Région bruxelloise, les écoles implantées à Bruxelles. Ainsi, alors que la segmentation catholique/laïc s'estompe sans disparaître, de nouvelles lignes de démarcation pourraient se cristalliser sur la base de proximité avec des champs connexes ou sur une base territoriale. La première d'entre elles n'est pas sans lien avec la perte d'autonomie de l'éducation vis-à-vis du champ économique. Ces lignes de démarcation émergentes semblent d'ores et déjà fragiliser le récent développement d'un véritable système éducatif.

Bernard Delvaux bernard.delvaux@uclouvain.be Université catholique de Louvain

Éric Mangez eric.mangez@uclouvain.be Université catholique de Louvain 
1 Cet article a été rédigé dans le cadre du projet de recherche intégré $\mathrm{n}^{\circ}$ 028848-2 financé par le $6^{\mathrm{e}}$ programme-cadre européen Know\&Pol (" The role of knowledge in the construction and regulation of health and education policy in Europe: convergences and specificities among nations and sectors »). Site internet : http://www.knowandpol. eu. La présente contribution s'appuie sur les études de cas réalisées en Belgique dans le cadre de cette recherche.

2 Durant l'année scolaire 1989-1990, le réseau libre (essentiellement catholique) scolarisait $69,6 \%$ des élèves de l'enseignement fondamental et secondaire en Communauté flamande et 47,2 \% de ces élèves dans les Communautés française et germanophone. Les effectifs des écoles gérées par les pouvoirs locaux représentaient quant à eux 15,5 et $34,7 \%$ des effectifs de ces Communautés. Actuellement, le réseau libre représente 67,2 \% de l'enseignement fondamental et secondaire en Communauté flamande et $49 \%$ en
Communauté française, tandis que les pouvoirs locaux représentent respectivement 16,9 et $36 \%$ des effectifs de ces Communautés (chiffres de 2011-2012 en Communauté flamande et de 2008-2009 en Communauté française).

3 Contre $57,5 \%$ pour la Communauté flamande et $0,5 \%$ pour la Communauté germanophone (OCDE, 1991, p. 35).

4 Puisque les Communautés n'ont pas de pouvoir fiscal.

5 L'enseignement qualifiant regroupe deux des filières (technique de qualification et professionnel) des quatre dernières années de l'enseignement secondaire ordinaire, certaines sections de l'enseignement secondaire spécialisé, les centres de formation en alternance ainsi que les formations en alternance des indépendants et des petites et moyennes entreprises.

\section{BIBLIOGRAPHIE}

BAKVIS H. (1985). " Structure and Process in Federal and Consociational Arrangements ». Publius, vol. 15, n², p. 57-69.

BYRNE S. (2001). "Consociational and Civic Society Approaches to Peacebuilding in Northern Ireland". Journal of Peace Research, vol. 38, n 3, p. 327-352.

CARVALHO L. M. \& COSTA E. (2009). Production of OECD's "Programme for International Student Assessment": final report. En ligne : www.knowandpol.eu/IMG/pdf/pisa. wp11.production-3.pdf (consulté le 12 mars 2013).

CATTONAR B., MANGEZ É., DELVAUX B., MANGEZ C. \& MAROY C. (2009). Réception, usage et circulation au niveau national d'un instrument supranational de régulation basé sur la connaissance : les enquêtes PISA. Le cas de la Communauté française de Belgique. Louvain-la-Neuve (Belgique) : CERISIS-GIRSEF.

CATTONAR B., DUMAY X., MANGEZ C. \& MAROY C. (2010). Évaluations externes dans l'enseignement primaire en Belgique francophone : réceptions et usages d'outils de régulation basés sur les connaissances. En ligne : http:// knowandpol.eu/IMG/pdf/belgium_educ_rapport_o3_ final_fr.pdf (consulté le 12 mars 2013)

DELVAUX B. (2011). "Connaissances et reconfiguration des entités de coordination. Le cas de l'enseignement en Belgique francophone ». Educação, sociedade \& culturas, no 34, p. 15-34.

DELVAUX B., MANGEZ C., MANGEZ É. \& MAROY C. (2008). The social and cognitive mapping of policy. The education sector in Belgium. En ligne : knowandpol.eu/IMG/pdf/ o1.belgiumeducation.pdf (consulté le 12 mars 2013).

De MUNCK J. (2002). "La Belgique sans ses piliers? Du conflit des modèles au choix d'une politique". Les Semaines sociales du MOC, "Piliers, réseaux et démocratie ", p. 95-115.

DUMONT H. \& DELGRANGE X. (2008). « Le principe de pluralisme face à la question du voile islamique en Belgique ". Droit et société, vol. 2008/1, n 68, p. 75108.

JADOT M. (2001). " L'évaluation administrative de la politique fédérale de l'emploi ". In C. de Visscher \& F. Varone (dir.), Évaluer les politiques publiques. Regards croisés sur la Belgique. Louvain-la-Neuve (Belgique) : Academia-Bruylant, p. 87-94.

JELEN T. G. \& WILCOX C. (1998). « Context and Conscience: The Catholic Church as an Agent of Political Socialization in Western Europe ". Journal for the Scientific Study of Religion, vol. 37, n० 1, p. 28-40.

LEMIEUX C. (2011). "Le crépuscule des champs. Limites d'un concept ou disparition d'une réalité historique? ". In M. de Fornel M. \& A. Ogien (dir.), Bourdieu, théoricien de la pratique. Paris: Raisons pratiques, p. 74-100.

LIJPHART A. (1979). " Consociation and Federation: Conceptual and Empirical Links ». Canadian Journal of Political Science / Revue canadienne de science politique, vol. 12 , n०3, p. 499-515.

LIJPHART A. (1985). "Non-Majoritarian Democracy: A Comparison of Federal and Consociational Theories ». Publius, no 15(2), p. 3-15.

MABILLE X. (2000). Histoire politique de la Belgique. Facteurs et acteurs de changement. Bruxelles : Ed. du CRISP (4e éd.).

MANGEZ C. (2010). "Les évaluations externes des acquis des élèves : description de dispositifs existants en Belgique francophone ". Les Cahiers de recherche en éducation et formation, n० 80 .

MANGEZ É. (2009). « De la nécessité de discrétion à l'État évaluateur ». La Revue nouvelle, n० 7-8, p. 32-37.

MANGEZ É. (2010) «Global Knowledge-based Policy in Fragmented Societies: the case of curriculum reform in French-speaking Belgium ". European Journal of Education, no $45(1)$, p. $60-73$. En ligne : http://dx.doi. org/10.1111/j.1465-3435.2009.01415.x (consulté le 12 mars 2013).

MANGEZ É. \& HILGERS M. (2012). « The Field of Knowledge and the Policy Field in Education: PISA and the production of knowledge for policy ". European Educational Research Journal, vol. 11, n², p. 189-205. En ligne : http://dx.doi.org/10.2304/eerj.2012.11.2.189 (consulté le 12 mars 2013).

MONNIER E. (2001). «État des pratiques d'évaluation dans les pays européens ". In C. de Visscher et F. Varone (dir.), Évaluer les politiques publiques. Regards croisés sur 
la Belgique. Louvain-la-Neuve (Belgique) : AcademiaBruylant, p. 55-64.

OCDE (1991). "Les systèmes éducatifs en Belgique : similitudes et convergences ». Bruxelles (Belgique) : Ministère de l'Éducation; OCDE.

REMAN P. (2002). "Quelques questions sur la société civile organisée ". Les semaines sociales du MOC, p. 4356.

SCHOON (2001). "L'évaluation des politiques publiques en Région wallonne : la vision de l'évaluateur ». In C. de Visscher \& F. Varone (dir.), Évaluer les politiques publiques. Regards croisés sur la Belgique. Louvain-laNeuve (Belgique) : Academia-Bruylant, p. 79-85.
SEILER D.-L. (1997). « Un système consociatif exemplaire : la Belgique ». Revue internationale de politique comparée, vol. $4, n^{\circ} 3$, p. 601-624.

VANDERSTRAETEN R. (2002). «Cultural Values and Social Differentiation: the Catholic pillar and its education system in Belgium and the Netherlands ". Compare, vol. 32, n², p. 133-148.

VARONE F \& de VISSCHER C. (2001). "Introduction ». In C. de Visscher \& F. Varone (dir.), Évaluer les politiques publiques. Regards croisés sur la Belgique. Louvain-laNeuve (Belgique) : Academia-Bruylant, p. 7-17.

VARONE F. \& JACOB S. (2004). « Institutionnalisation de l'évaluation et nouvelle gestion publique : un état des lieux comparatif ". Revue internationale de politique comparée, vol. 11, no 2, p. 271-292. 